\title{
Research and Design of Energy Data Acquisition System in Cement Enterprise
}

\author{
Xiangsong $\mathrm{Li}^{1, \mathrm{a}^{*}}$, Xiaohong Wang ${ }^{1, \mathrm{~b}}$ and Shaohong Jing ${ }^{1, \mathrm{c}}$ \\ ${ }^{1}$ School of Electrical Engineering University of Jinan, Jinan 250022, China \\ a15063342010@163.com, b cse_wxh@ujn.edu.cn, ${ }^{c}$ cse_jsh@ujn.edu.cn
}

\section{Keywords: Cement Production,Energy Management,Data Acquisition.}

Abstract. In the growing demand for energy, while energy supply is becoming increasingly tense, prices continue to rise. This makes the energy consumption cost plays a more and more proportion in the enterprise cost calculation. To improve the energy utilization rate, cement enterprises take various optimization reconstruction and energy conservation plan. To begin with, we need to find processes with unreasonable energy utilization. This needs for statistical analysis for energy consumption status of enterprises and search for energy-saving potential of each link.

Based on the cement production process as the research object, self-developed data acquisition system, determine which energy data need to collect in the complete cement production line and these data collection, classification and preliminary processing, provides the data support for the cement enterprise energy management system, provides basic information for the statistics, analysis and forecast of the power consumption of cement enterprises, coal and other resources, so that enterprises have an intuitive understanding of their energy consumption, find out the weak link of the enterprise, reduce costs, reduce energy consumption and improve economic performance. And provide the theoretical basis and objectives for the enterprise to develop energy saving plan.

\section{Introduction}

China's national conditions about the energy issues is that energy utilization rate is low and energy wastage is serious. The factors that affect the energy consumption of the enterprise occur in every link of the production process, and complex interweave together, therefore, it is difficult to accurately estimate the influence of various factors on energy consumption. Only by means of scientific, effective and quantitative analysis system can we catch the key point of saving energy and reducing consumption and make reasonable decisions.

Cement enterprise energy management system is a comprehensive monitoring and rational analysis of the energy consumption trend and energy utilization level of the production process. Based on the energy consumption statistics and energy consumption monitoring, Operators can search the energy-saving potential, develop energy-saving measures, in accordance with changes in the operating parameters in a timely manner to all kinds of energy consumption of scheduling, so that the energy consumption is more reasonable, so as to reduce energy consumption, lower production cost. As a subsystem of the energy management system, data acquisition system can first collect the data in real time so as to reflect the energy consumption status of all the monitoring points that distributed in each production process accurately and rapidly, then make analyzation, classification and processing of the data.[1-2]

\section{System Design}

Intruction of the design of this data acquisition system was based on a branch company in Baiyin City which belongs to Ningxia building materials group Co.,Ltd.This branch company adopted NSP cement production line and had a $4500 \mathrm{t} / \mathrm{d}$ production line of clinker, a $9 \mathrm{WM}$ production line of cogeneration, two $\Phi 4.2 * 13 \mathrm{~m}$ production lines of cement.

Data sources of the energy data were intelligent instruments, DCS (Distributed Control System) and existing softwares in the company such as the ERP. Different kinds of data sources that scattered in 
different locations were integrated into data acquisition system by computer network technology and bus technology and the function of data acquisition, production monitoring, material-balance calculation, energy consumption calculation and alarm recording were realized by the software of data acquisition system.

Production Processes of the Cement.NSP cement production process could be divided into three main working procedures. The first is preparation of raw meal and it contains a number of processes such as crushing, prehomogenizing, blending of the material and grinding, prehomogenizing of the raw meal. The second is clinker burning and it contains a number of processes such as grinding, conveying of the coal and preheating, decomposing and cooling of clinker. The last is preparation of cement and it contains a number of processes such as storing, blending of clinker and grinding, storing and packing of cement.[3]

Analyzation of the Data in the System.Data flows of this company in energy consumption are as shown in the Figure 1. Data sources of this system were divided into five categories. Of these, the electricity data and production data were provided by ammeters mounted on serial device servers, DCS, existing softwares and manual data entry and the operation of the system itself produced operating data. With the use of comparison table, system would get different kinds of data in different methods and then divide the data into five preliminary categories. Then the data were divided into thirteen particular categories for data processing. The outcome of the data processing were data for production monitoring, various kinds of reports, warning messages and operation record of the system.

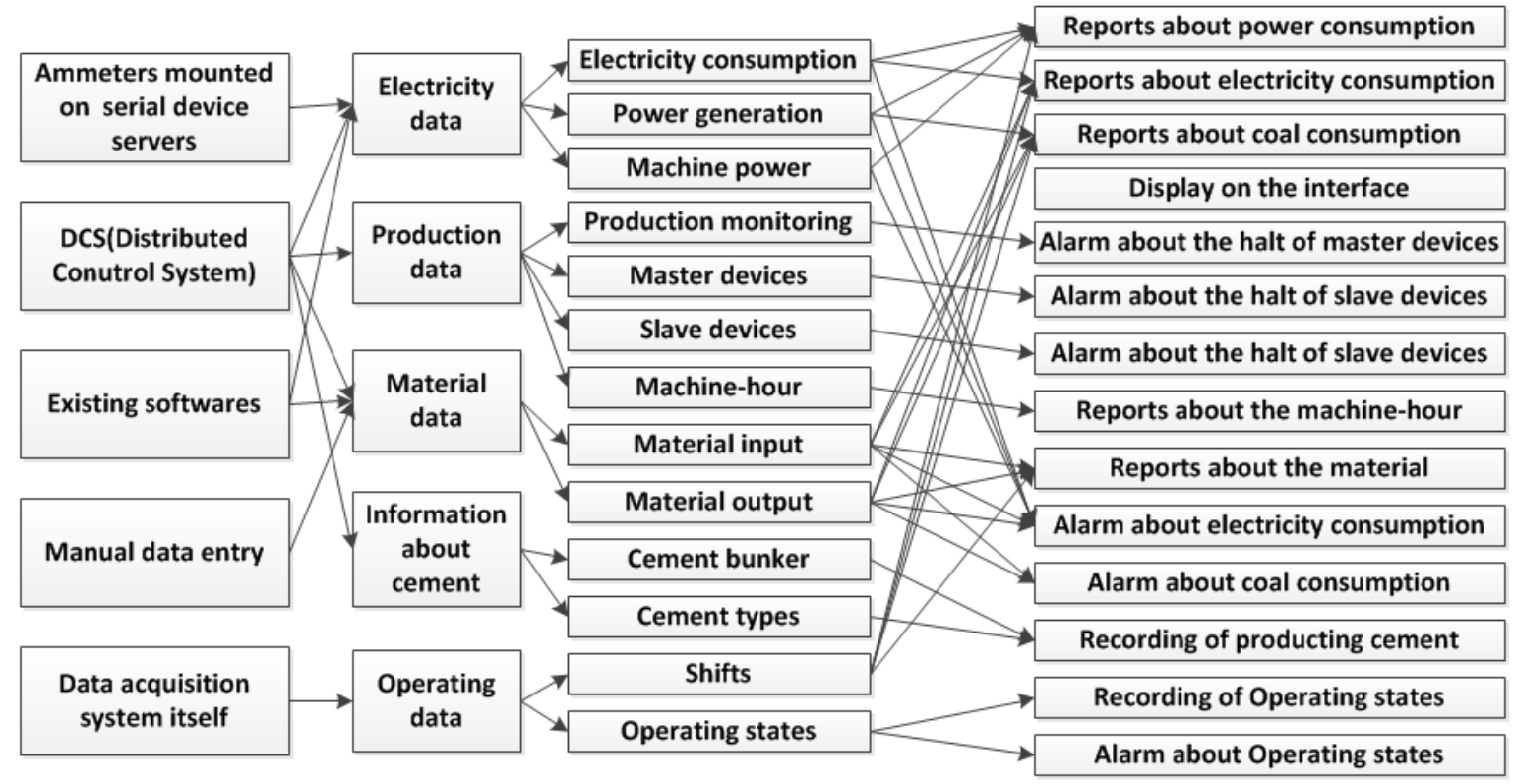

Fig.1. Data flows of the company in energy consumption

The selection of monitoring points. The energy used in the process of cement production includes : electricity, coal, gasoline, diesel oil and water. Electricity and coal's consumption accounted for $98.72 \%$ of the total energy consumption of cement manufacturing in 2006 [4]. Thus the electricity and coal are the major energy consumption of cement manufacturing and they are the main research object of this paper.

Electricity monitoring points. The selection of electricity monitoring points based primarily on the following principles[5]:

a) Total input-wire and incoming and outgoing line of step-down converter in general step-down station.

b) Incoming and outgoing line of transformers and feeding place of high voltage motors in workshop transformer substations.

c) Low voltage equipment of working procedures and standalone devices in electrical rooms. 
Because of the diversity of process design and electrical design of the cement production line, many factors were considered, such as supplying and distributing electricity lines, power supply and distribution equipment and metering equipment of electricity and material. Then decisions like whether the position and quantity of existing electricity monitoring points were appropriate, how to place the newly added monitoring points and how to build the new communication network of newly added monitoring points were decided.

Coal monitoring points. The input-output of coal are in production processes of coal mill, decomposing furnace and rotary kiln and DCS will monitor those points. So those information can be obtained from the DCS.

Design of the hardware network. The design of the hardware network is shown in Figure 2.

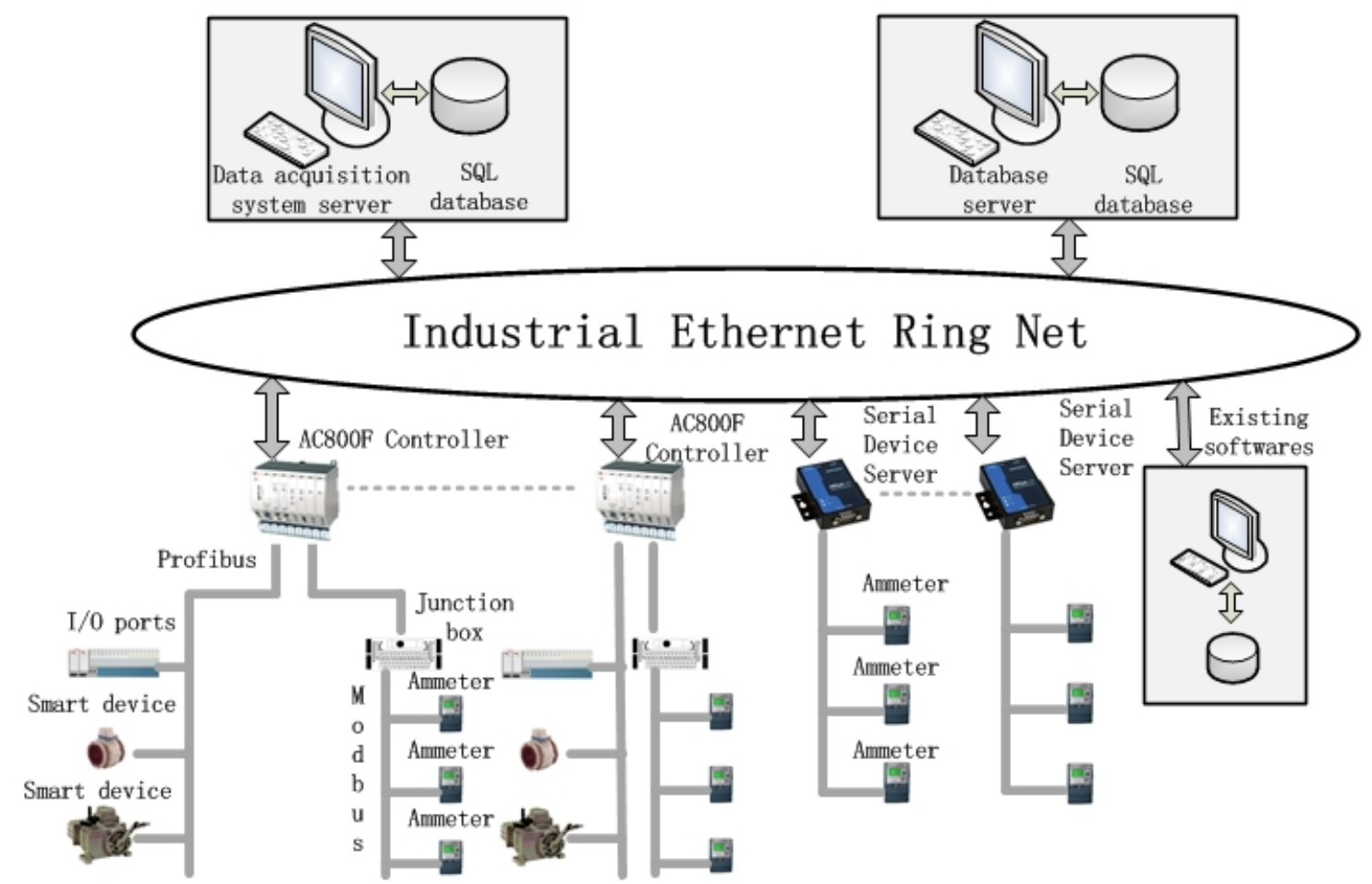

Fig.2. Design of the hardware network

By using OPC standard interface, system could acquire operating state and energy consumption information of the major energy consumption equipment, input-output of the material, etc. The DCS of this branch company is Freelance process control system of $\mathrm{ABB}$ and the model of controller is AC800F. Controller communicates with I/O ports and smart devices through Profibus protocol, and communicates with ammeters through Modbus protocol.

Electrical energy consumption is mainly through the serial network solitary network acquisition. Ammeters are connected by electrical connection in parallel and accessed to serial device servers through RS485 interface. Serial device servers connect RS-232 / 485 / 422 serial devices to the TCP / IP network, to achieve data bidirectional transmission of RS-232 / 485 / 422 serial and TCP / IP network interface.

\section{Design of the software.}

Function of parallel table. To reduce the coupling degree with other systems and improve the generality and maintainability, data acquisition system adopted technique of parallel table. There are two kinds of parallel tables: parallel table of ammeters and parallel table of DCS.

Parallel table of ammeters. Data sources of electricity are ammeters in DCS, ammeters in serial communication network and monitoring points of existing software. There are many fields to describe basic information, data source and attainment method of the monitoring points detailed and its shown 
in Table 1.By using parallel table of ammeters, information about monitoring points sent to relevant acquisition modules and then data in acquisition modules are summarized and sent to data processing module. For example, after acquiring communication protocol, contact address and CommPort, relevant acquisition module could create and send the command frame, acquire and decode the response frame. Then data of electricity are summarized and sent to electricity array variables so as to process the data, generate reports, generate alarm message, etc. To ensure the low coupling degree, each data source system is independent of others and each acquisition module runs in parallel. This system is suitable for cement enterprises with different data sources of electricity and it allows different data sources exist at the same time, these two kinds of advantages make the system more universal. Through configuration of the parallel table of ammeters, rather than modification of the software, ammeters' quantity and information can be modified. This makes maintenance of the system easier.

Table 1. Parallel table of ammeters

\begin{tabular}{|c|c|c|}
\hline Classification & Field Name & Description \\
\hline \multirow{7}{*}{ Basic information } & AmmeterNumber & The serial number of the meter \\
\hline & AmmeterName & The name of the meter \\
\hline & AmmeterSource & Data sources \\
\hline & ElectricRoom & Electric room that meter belongs to \\
\hline & $\mathrm{CT}$ & Current Transformer \\
\hline & PT & Phase voltage Transformers \\
\hline & $\ldots \ldots$ & $\ldots \ldots$ \\
\hline \multirow{3}{*}{$\begin{array}{l}\text { Felds for ammeters in } \\
\text { serial communication } \\
\text { network }\end{array}$} & CommunicationProtocol & Communication protocol of the meter \\
\hline & AmmeterAddress & Slave Address \\
\hline & CommPort & Comm port \\
\hline \multirow{4}{*}{$\begin{array}{l}\text { Felds for ammeters in } \\
\text { DCS }\end{array}$} & IpAddress & $\begin{array}{l}\text { IP of the OPC server that meter belongs to } \\
\text { Name of the OPC server that meter belongs }\end{array}$ \\
\hline & OPCName & to \\
\hline & ElectricEnergyItem & $\begin{array}{l}\text { Name of the OPCItem to acquire } \\
\text { electricenergy }\end{array}$ \\
\hline & $\ldots \ldots$ & ..... \\
\hline \multirow{4}{*}{$\begin{array}{l}\text { Felds for ammeters in } \\
\text { existing softwares }\end{array}$} & & Database table name of Demand Side \\
\hline & ElectricEnergyTableNameDSM & Management to acquire electricenergy \\
\hline & & Database field name of Demand Side \\
\hline & $\begin{array}{l}\text { ElectricEnergyFieldNameDSM } \\
\ldots . . .\end{array}$ & $\begin{array}{l}\text { Management to acquire electricenergy } \\
\ldots . . .\end{array}$ \\
\hline
\end{tabular}

Parallel table of DCS.Multiple sets of DCS exist in a large-scale enterprise concurrently and data under the same DCS can be divided into many types such as condition monitoring-data, master and slave machines, machine-hour, input and output of the material, etc. After acquiring the data through OPC communication, classification of those complicated data is based on parallel table of DCS.As shown in Table 2, parallel table of DCS contains basic information and classified information. According to the basic information, system can define the OPC Server, OPC Group, OPC Item and obtain the required variables' value. Then those data are classified and managed on the basis of classified information. Take the master device for example, "MasterOrSlave" is the field to show whither this variable is a running signal of master device or slave device in DCS," HaltID" is the field to show the id so as to find detailed information of the master device in table of master devices. After getting the above information, we can monitor the running state and generate reports. Through configuration of the parallel table of DCS, variables can be added, modified and deleted. This makes the system more universal, maintenance more convenient. 
Table 2. Parallel table of DCS

\begin{tabular}{cll}
\hline Classification & Field Name & Description \\
\hline & ID & Primary Key \\
& VariableName & Variable Name \\
& VariableDescription & Variable Description \\
Basic information & & Name of the OPC server that variable \\
& OPCName & belongs to \\
& Item & Name of the OPCItem \\
& $\ldots \ldots$ & $\ldots \ldots$ \\
\hline \multirow{2}{*}{ Felds for data } & ViewName & View name that variable belongs to \\
classification & MasterOrSlave & Flag of cumulant \\
& $\ldots . .$. & Flag of master machine and slave machine \\
& & $\ldots \ldots .$. \\
\hline
\end{tabular}

Data storage.Database platform is SQL Server 2012 which is relational database management system and the interface is OLEDB.

Data acquisition system contains four kinds of database: management database, branch database, DCS database and cache database. Each branch has its individual management database and branch database. Management database is used to store basic parameters of data acquisition system, reports, running logs and alarm logs. Branch database is used to store gathered initial data and calculated intermediate data. Because of the short cycle-time and large quantity, every DCS has its own database to store real time data and historical data. There is a one-to-one correspondence between cache database and data acquisition software and both of them are collocated in the same acquisition server. When the network connections is disconnected, cache database is used to store the data manipulation instructions to other databases. When the network connections is connected, data manipulation instructions in cache database will be executed.

\section{Conclusions}

The data acquisition system had fully considered the complex industrial environment and diversified acquisition objects and completed the function of the expected with strong universality and high extensibility. System has been put into use in a number of cement companies, the system is running stable, and the effect is obvious.

\section{Acknowledgements}

This work was financially supported by Major projects of Shandong Province independent innovation achievements (2014CGZH0601) and China-EU SMEs Cooperation Fund for energy conservation research project (SQ2013ZOC600003).

\section{References}

[1]Taoping Li. Energy consumption status and report on energy saving potential about China's cement industry[J]. Cement Engineering,2004,(04):1-6,In Chinese

[2]Xiaobo Wang. The research of electric furnace production process energy consumption monitoring andintelligent analysis methods of power consumption[D]. Changchun :Changchun University Of Technology,2012:1-4,14-20, In Chinese

[3]Zhijiang Liu. Technology of NSP Cement Production[M].beijing: China Building Materials Press,2005, In Chinese 
[4]Hongjin Zhou. Commentary on energy consumption of cement industry in 2006[J].China Cement,2006,(10)26-29, In Chinese

[5]GB/T 27977-2011,Test and calculation methods of electricity efficiency of cement production[S] 